\title{
EDUCACIÓN PARA LA SALUD EN LAS FAMILIAS INMIGRANTES MARROQUÍES
}

\author{
Isabel Gentil García
}

Diplomada en Enfermería. Doctora en Antropología Social y Cultural. Profesora E. U. Enfermería, Fisioterapia y Podología. Universidad Complutense de Madrid

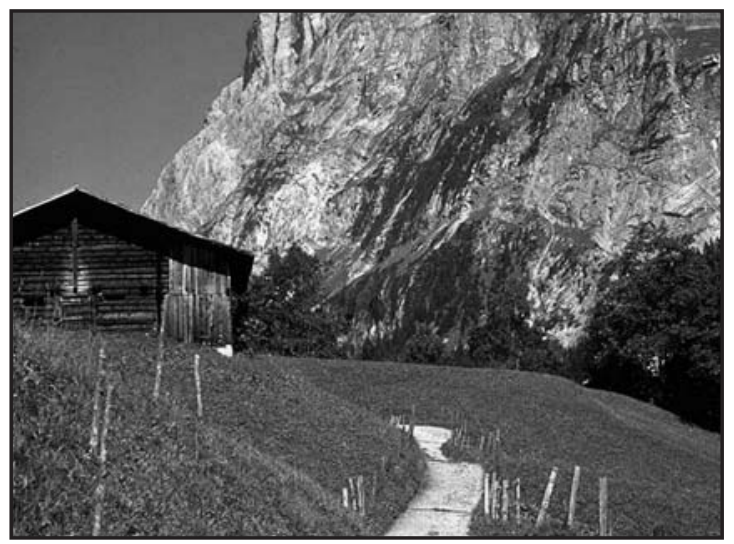

\section{EDUCATION FOR HEALTH IN MOROC- CAN IMMIGRANT FAMILIES}

\section{SUMMARY}

$\mathrm{T}$

To effectively carry out Health Education it is necesary to know the characteristics of the population it is aimed for. The Transcultural Nursing Model (M. Leininger) sustains that it is necessary to know the values and beliefs of other cultures to provide competent care .We are all born and raised in the heart of a certain cultural tradition that "makes us ". Every culture creates its models on the ideal way of being which tell us how to behave or not. For all this, the most characteristic features that identify the model of a good Muslim are presented. Taking in consideration that these people are immigrant Muslims who live in a social and cultural context different from that of origin. How this situation can be of influence will be analyzed . Once the most common characteristics are known, some skills to be competent in Health Education for the immigrant Moroccan families will be described.

Keywords: Nursing. Moroccan immigrants. Education for health.
EDUCAÇÃO PARA A SAÚDE NAS FAMÍLIAS IMIGRANTES MARROQUINAS

\section{RESUMO}

Dara realizar ação educativa para a Saúde de forma eficaz é imprescindível conhecer as 1 características da população a que se dirige. O Modelo de Enfermagem Transcultural (M. Leininger) afirma que é necessário conhecer valores e crenças de outras culturas para proporcionar cuidados competentes. Todos nascemos e crescemos no seio de uma determinada tradição cultural. Cada cultura credita seus modelos sob forma de ser ideal, servindo para nortear como devemos nos comportar em determinadas situações. Sendo assim, apresentam-se perfis mais característicos que identificam o modelo cultural mulçumano. É preciso considerar que pessoas muçulmanas imigrantes vivem em um contexto social e cultural diferente daquele de onde provêem. Este trabalho analisará como se dá a influência cultural nesta situação. Ademais, uma vez conhecidas as características mais comuns dessa população, serão descritas algumas habilidades no tocante ao Ser Competente para a Educação em Saúde voltada às famílias de imigrantes provenientes de Marrocos.

Palabras- chave: Enfermagem. Imigrantes marroquinos. Educação para a saúde.

\section{RESUMEN}

$\mathrm{P}$ ara realizar Educación para la Salud de forma eficaz es imprescindible conocer las características de la población a la que va dirigida. El Modelo Enfermería Transcultural (M. Leininger) afirma que es necesario conocer valores y creencias de otras culturas para proporcionar cuidados competentes. $Y$ es que todos nacemos y crecemos en el seno de una determinada tradición cul- 
tural que "nos hace". Cada cultura crea sus modelos sobre la forma de ser ideal que sirven para saber cómo debo comportarme o cómo no debería comportarme. Por todo ello se presentarán los rasgos más característicos que identifican el modelo de buen musulmán. Teniendo presente que estas personas son musulmanes inmigrantes que viven en un contexto social y cultural diferente al de procedencia. Se analizará cómo puede influir ésta situación. Una vez conocidas las características más comunes se describirán algunas habilidades para ser competentes en Educación para la Salud a familias inmigrantes marroquíes.

Palabras clave: Enfermería. Inmigrantes marroquíes. Educación para la salud.

\section{INTRODUCCIÓN}

Se entiende Educación para la Salud a un proceso de comunicación interpersonal que capacita a las personas para la toma de decisiones en relación a problemas de salud (OMS, 1989).

Antes de iniciar el proceso es imprescindible conocer las características de la población a la que va dirigida. En este caso ¿qué conocemos de los inmigrantes marroquíes? Algunos datos (INE, 2008): en enero de 2008 eran la población inmigrante más numerosa en España, en concreto 582.923 , lo que representa el $12^{\prime} 9 \%$ del total de inmigrantes. La mitad de ellos con autorización de residencia permanente. Que en su país de procedencia la población es muy variada en etnias: árabes y diferentes etnias bereberes, que se hablan diversas lenguas: árabe marroquí, lenguas bereberes: amazight, tarifit, rifi coloquial, dhamazight, que es variada también su lugar y forma de residencia: $55^{\prime} 2 \%$ de la población es urbana y el 44'8 rural. Los que han emigrado proceden sobretodo del ámbito rural y son bereberes, además es este grupo poblacional el que mayor choque cultural va a experimentar. Todos estos datos nos informan de cuántos son, quiénes son, de dónde proceden, pero no nos dicen nada de cómo son, para ello necesitamos datos cualitativos.

El modelo de Enfermería Transcultural de M. Leininger (1999) afirma que es necesario conocer valores y creencias de otras culturas para proporcionar cuidados competentes. Y es que cada uno de nosotros nacemos y crecemos en una determinada tradición cultural que nos hace, nos hemos construido dentro de una determinada cultura. Cada cultura crea modelos sobre la forma de "ser ideal", modelos en los que las personas nos reconocemos y que sirven para saber cómo debemos comportarnos o cómo no deberíamos comportarnos. Aunque no todos los pertenecientes a una cultura piensan exactamente igual y de la misma manera pues los humanos no somos clones y existe la diversidad dentro del grupo, pero también no todos piensan de modo totalmente diferente. Es el Islam lo que proporciona una identidad común a los marroquíes y dicta los valores y normas de conducta.

Pero antes de describir los rasgos más característicos del ser musulmán, un apunte para incitar a la reflexión. Un inmigrante me decía, para que oyeran mi respuesta otros inmigrantes: “¿Verdad que en España todos los días, en todas las casas se cena tortilla de patata?" Bueno, ¡todos los días y en todas las casas! es mucho afirmar, aunque sí es cierto que la tortilla de patata es parte de nuestra identidad colectiva. Esta anécdota pretendo que sirva para apoyar que debemos huir de las universalizaciones, son erróneas siempre, y ser muy cautos con las generalizaciones porque pueden equivocarnos al oscurecer los matices, los detalles, y precisamente en ellos es donde se encuentra lo más importante de nosotros. Quiero insistir que las generalizaciones de las que voy a hablar, construidas con los rasgos más frecuentes en el modelo de ser musulmán, debemos percibirlas con carácter analítico. Necesitamos hacer clasificaciones para no perdernos en el universo de los datos, de los atributos. Estas generalizaciones son el punto de partida para poder seguir avanzando. Hacer hincapié y recalcar que las generalizaciones no son finales: así son todos los marroquíes punto final. Sino todo lo contrario. Con los rasgos más comunes o que más se repiten confeccionamos una generalidad inicial que nos sirve para a partir de ahí seguir profundizando.

\section{ENTORNO SOCIAL Y FAMILIA}

La sociedad musulmana es una sociedad vertical y jerarquizada. Islam significa sumisión. El poder, la autoridad, los mandatos vienen de arriba abajo. Alá, El Corán, el rey, los juristas que son 
también teólogos, los ancianos, los hombres, las mujeres, los niños. La actitud hacia el superior debe ser de sumisión.

Hay dos valores fundamentales. Uno es la solidaridad familiar. Otro la solidaridad del grupo de parientes. Es un principio básico que los primeros en llegar a España desarrollen una red de acogida para los que llegan después, por esto veremos como en los asentamientos de marroquíes en las ciudades de España están agrupados por el lugar de procedencia en Marruecos. Esto contribuye a mantener los vínculos entre la sociedad de origen y de destino. Recordemos que la solidaridad es factor de protección para la salud. "A mayor solidaridad y sociabilidad más salud." (Navarro, 2008). Las redes familiares, de amistad y del mismo pueblo refuerzan el sentimiento de pertenencia, pero también van a actuar como mecanismo de control, haciendo que los individuos se comporten de forma respetuosa con los valores musulmanes.

Otros valores importantes son el honor y el respeto. El honor se demuestra obedeciendo y siendo respetuoso con las normas y valores de la familia y comunidad. El comportamiento no adecuado de un miembro de la familia es una ofensa para toda la familia. El respeto es sobre todo a los mayores, a los ancianos. Se considera que ellos son conocedores de la vida, cómo han vivido más es lógico que sepan más y por tanto que los jóvenes e inexpertos les obedezcan y respeten.

\section{¿Cuál es el modelo de buena musulmana?}

Principalmente ser una mujer decente, decente y respetada, que se ocupa y preocupa por las cosas de mujeres, las del espacio interior privado, frente al hombre que se ocupa y preocupa del espacio exterior, el espacio público. La decencia en una mujer incluye el no mostrar el cuerpo, en no hacer nada que pueda considerarla como seductora o provocativa para los hombres. Tiene que ser modesta en su apariencia externa cuando está en la calle. Debe saber hacerse respetar. El uso del velo es una práctica de ocultamiento corporal a los hombres que no son el esposo. En casa se lo quitarán ante un reducido número de familiares directos. Muchas pueden sentirse "desnudas" sin el velo. Las mujeres llevan el peso del honor de la familia y de cada uno de los miembros de la familia, de tal forma que la mujer no decente no es que se degrade ella, es que degrada a todos y a la familia en conjunto.

Esta concepción del cuerpo como algo que debe permanecer oculto, inculcado desde la infancia, extendido y mantenido por la sociedad hace que el pudor a mostrar el cuerpo desnudo sea algo realmente importante en ellas. De aquí lo delicado que les resulta ser exploradas por profesionales de la salud hombres. Nosotros, como profesionales de la salud, no somos quien para juzgar o valorar estas costumbres, sino que debemos conocerlas para poder practicar cuidados competentes. Debemos reflexionar ¿qué queremos? demostrar nuestra "superioridad occidental" o prestar cuidados competentes. Hay mujeres musulmanas que perciben, y no lo entienden, que causan pena y compasión en el mundo occidental, hay otras que sufren la humillación de ser objeto de burla, y tampoco lo entienden.

Las mujeres mayores buscan tener la seguridad de la lealtad de sus hijos varones para la vejez y suelen dominar a las jóvenes, manteniendo y fortaleciendo la situación de dominio del esposo sobre la esposa.

\section{¿Cuál es el modelo de buen musulmán?}

Ser trabajador. Ser la persona que proporciona el sustento económico. Sus valores son tener fortaleza física, no mostrar debilidad en ningún aspecto, no estar enfermo. Está desvalorizado el hombre enfermizo. Debe de ocuparse y preocuparse de las cosas del espacio exterior. Dentro del matrimonio tiene el papel de director del mismo; legalmente es así como queda recogido en el "Código marroquí del Estatuto de Personas" o Moudawuana, que define el matrimonio como un contrato legal mediante el cual un hombre y una mujer se unen para llevar a cabo una vida conyugal común y duradera, bajo la dirección del marido. (Motilla, 2002)

Por todo lo anterior nos encontramos, a veces, con que es el marido quien habla por la mujer. Como profesionales de la salud debemos conocer las características culturales de las personas a quienes prestamos cuidados para ser competentes culturalmente.

\section{Los hijos}

Las relaciones de los hijos con los padres son de respeto y obediencia, derivado de la autoridad 
paterna. Me contaba un rifeño de 23 años que él, en el Rif, cada día cuando llegaba a casa besaba la mano de su padre, “y ¿a tu madre?", le pregunté yo, "a ella le doy un beso en la frente".

El niño marroquí debe esforzarse y prepararse para trabajar fuera de casa. Si es niña, además de trabajadora y aprender las cosas de la casa, sobretodo ser decente.

El rol de la madre para con los hijos es el de cuidadora, y mantenedora y trasmisora de los valores de la tradición. Dentro del papel de cuidadora está el conocimiento de plantas con propiedades curativas. Es un conocimiento propio de las madres y que se transmite de generación en generación. El rol del padre para con los hijos es el de guía, y como buen guía se da por sentado que es él quien conoce el camino y la dirección. Al padre se le reconoce el poder, el derecho y la obligación de sancionar a sus guiados si considera que están en riesgo de extraviarse, incluido el castigo físico. Como profesionales comprometidos con la defensa de la salud es importante conocer para entender (que no justificar) cómo se construye la lógica interna que lleva a ello, porque este conocimiento es lo que nos va a permitir actuar para modificar actuaciones que dañan la salud.

\section{LOS INMIGRANTES ANTE LA NUEVA SOCIEDAD}

El inmigrante está dotado de conocimientos, habilidades y estrategias para desenvolverse en el medio social de donde procede, pero en la nueva sociedad se encuentra con prácticas sociales que

\section{La difícil situación de los hijos}

Los valores y costumbres que le enseñan en la familia son diferentes a los que aprende en el espacio exterior: barrio, amigos, escuela. Lo viven como conflicto, pues se identifican con valores y costumbres de la nueva sociedad pero les duele tener que enfrentarse a sus padres, son conscientes de los sacrificios que ellos hacen y han hecho, del cariño que les tienen -ellos también quieren a sus padres-.

Si los adolescentes, en general, se encuentran en un espacio indefinido entre la niñez y la adultez, el adolescente marroquí además, está en un no espacio; ni de aquí, ni de allí, lo que hace que a veces en el ambiente familiar se definen como españoles y en entre los autóctonos como marroquíes. Como profesionales de la salud no ignoraremos esta vivencia de conflicto y cuando tengamos que darles recomendaciones en educación para la salud lo haremos explicando la lógica interna de la recomendación. Son especialmente receptivos y con ganas de aprender, aunque aparentemente por la situación que están experimentando dé la impresión contraria. No caigamos en el desánimo.

A veces los padres les exigen demasiado a estos niños porque se pone en ellos excesivas expectativas. Que vayan a la Escuela para poder tener un empleo mejor que el del padre, "quiero que mis hijos puedan tener trabajos bajo techo", me comentaba un marroquí que trabaja en la construcción. Pero los padres desconocen la fuerte competitividad y que el punto de partida de estos niños no suele ser desde el mismo nivel que el de los autóctonos. También puede ser lo contrario, las prisas para que se pongan a trabajar, no entendiendo que con 16 años (en su concepto un adulto) aún sea obligatorio el estar escolarizado y tener prohibido trabajar según la legislación española.

\section{REFUERZO DEL PROPIO CAPITAL CULTURAL}

Para garantizar la supervivencia en un entorno desconocido y adverso, los inmigrantes adultos, y más si son las madres de familia que tienen menos interrelación con el mundo de fuera del hogar, tienen que recurrir a su propio capital cultural y simbólico: el Islam, reforzándolo.

El Islam da consuelo, protección y seguridad frente a un ambiente que se percibe como hostil. El Islam refuerza la identidad para resistir mejor las condiciones difíciles que acompañan al desarraigo en todo inmigrante. Revaloriza la identidad tras la desvalorización de lo musulmán en el mundo occidental. En definitiva es la liberación de unas condiciones de vida realmente duras. Cuanto más miedo hay a no ser, más nos refugiamos en el modelo de cómo se debe ser, sobre todo en los rasgos externos, observables. Es una forma de protegerse frente al ambiente adverso. Son estrategias para sobrevivir en situaciones desfavorables. Recordar que quien más sufre dentro del amplio fenómeno de la emigración es el emigrante, sobre todo en sus comienzos, cuando además se acompaña de situaciones económicas precarias. La exclu- 
sión social conduce a refugiarse en la religión, el espacio sagrado que salvaguarda de todo lo malo mundano y consuela con el bien eterno.

\section{COMPETENCIA EN EDUCACIÓN PARA LA SALUD}

\section{Aspectos a tener en cuenta:}

- No hacer juicios de valor, no somos jueces, somos profesionales de la salud que pretendemos ser competentes culturalmente. M. Leininger (1994), dice que la imposición y el etnocentrismo por parte del personal de enfermería reducen en gran medida el descubrimiento de conocimientos y, consecuentemente la calidad de los cuidados.

- Desarrollar capacidad de inspirar confianza a través de la capacidad para entender los rasgos culturales y valorar la diversidad. A. Malouf (2001) apunta que cuanto más perciba el inmigrante que se respeta su cultura de origen, más se abrirá a la cultura del país de acogida.

- El microespacio. La distancia física interpersonal en la interacción con personas musulmanas es diferente a como lo percibimos. Este espacio o burbuja de seguridad es mayor especialmente entre personas de distinto sexo.

- La mirada. El respeto hacia el que se considera superior implica no mirarle a los ojos. Me comentaba una maestra en relación a un niño musulmán: "Es que cuando le hablo no es capaz de mirarme a los ojos, siempre mirando al suelo" Es lo que le han enseñado.

- Ayuno del mes de Ramadán (Quingles, 2002). Personas a las que obliga el ayuno: adulto, estar en su sano juicio, tener capacidad y fortaleza física. Personas exentas de la obligación de ayunar: niños, enfermos, viajeros, ancianos, embarazadas, mujeres lactantes y mujeres en período menstrual. Pero muchas de las personas exentas prefieren practicar el ayuno por religiosidad o por otras razones, como formar parte del colectivo que tiene las características de las personas con obligación. Acciones permitidas: Tomar algún medicamento siempre que no llegue al estómago. Inyectarse medicamentos que no sean sustitutivos de alimento.

- No están acostumbrados a las preguntas directas, a responder a cuestionarios estructurados rígidos, a pasar de una cuestión a otra con rapidez, es mejor entrevistas charlas pausadas, sin prisas.

A pesar de la importancia dada al conocer para ser competentes, ahora que ya conocemos anteponemos a la aptitud la actitud. La capacidad para ser competentes en cuidados se inicia con la capacidad para la empatía. La empatía la define Howard Gardner (2005) como la capacidad intelectual de una persona de vivenciar la manera en que siente otra persona y de compartir sus sentimientos, lo cual puede llevar a una mejor comprensión de su comportamiento o de su forma de tomar decisiones. Es la habilidad para entender las necesidades, sentimientos y problemas de los demás y responder correctamente a sus reacciones emocionales. Por tanto desarrollar competencia en Cuidados implica desarrollar competencia emotiva, es desarrollar una sensibilidad y ante todo una actitud de respeto hacia la persona. Los humanos estamos dotados de capacidad para entendernos. Debemos buscar ¿qué nos une?: la necesidad de cuidados. Esta necesidad es universal. Desnudos del traje cultural, todos los humanos sentimos igual, nos emocionamos igual, amamos, sufrimos, gozamos igual.

\section{BIBLIOGRAFÍA}

- Gardner H. (2005) Las cinco mentes del futuro: un ensayo educativo. Paidós, Barcelona.

- INE. Enero 2008. Avance del padrón de 2008

- Leininger M. (1994) Teoría de los cuidados culturales. En Marriner-Tomey. Modelos y teorías en enfermería. Doyma, Madrid.

- Leininger M. (1999) Cuidar a los que son de culturas diferentes requiere el conocimiento y las aptitudes de la enfermería transcultural. Cultura de los Cuidados. Revista de Enfermería y Humanidades 6, 5-8.

- Maalouf A. (2001) Identidades asesinas. Alianza Editorial, Madrid.

- Navarro V. (2008) El País. 1 mayo 2008.

- Motilla A, Lorenzo P. (2002) Derecho de Familia Islámico. Problemas de adaptación al Derecho español. Colex, Madrid. - Organización Mundial de la Salud. (1989) Educación para la Salud: manual sobre educación sanitaria en atención primaria de salud. OMS, Ginebra.

- Quingles J. (traductor, prólogo y notas) (2002). Dichos del Profeta: los cuarenta hadices. Al-Nawawi. José J. de Olañeta, Palma de Mallorca. 